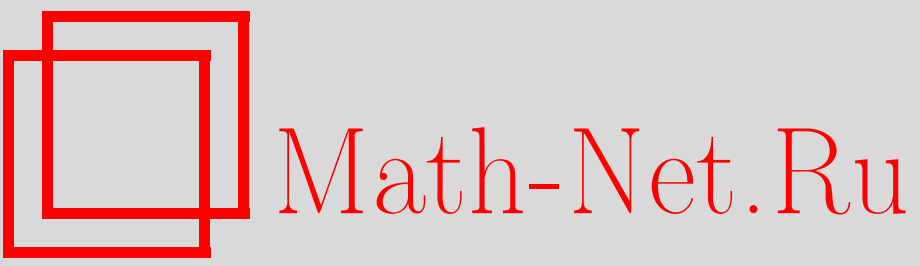

Ю. В. Муранов, Д. Реповш, Геометрические свойства спектральной последовательности теории перестроек, УМН, 2002, том 57, выпуск 6, 191-192

DOI: https://doi.org/10.4213/rm587

Использование Общероссийского математического портала Math-Net.Ru подразумевает, что вы прочитали и согласны с пользовательским соглашением

http://www . mathnet.ru/rus/agreement

Параметры загрузки:

IP : 54.89 .56 .158

26 апреля 2023 г., 12:03:09 


\title{
ГЕОМЕТРИЧЕСКИЕ СВОЙСТВА СПЕКТРАЛЬНОЙ ПОСЛЕДОВАТЕЛЬНОСТИ ТЕОРИИ ПЕРЕСТРОЕК
}

\author{
Ю. В. Муранов, Д. РеПовш
}

Пусть $X$ - замкнутое топологическое многообразие размерности $n \geqslant 5$ с фундаментальной группой $G=\pi_{1}(X)$, имеющей подгруппу $\pi \subset G$ индекса 2 . Рассмотрим отображение $\chi: X \rightarrow$ $\mathbb{R} P^{m}$ многообразия $X$ в $m$-мерное действительное проективное пространство высокой размерности. Пусть $\chi$ индуцирует эпиморфизм фундаментальных групп $\chi_{*}: G \rightarrow \mathbb{Z} / 2$ с ядром $\pi$. Обозначим через $Y$ одностороннее подмногоообразие $X$, которое является трансверсальным прообразом $\chi^{-1}\left(\mathbb{R} P^{m-1}\right)$ одностороннего подмногообразия $\mathbb{R} P^{m-1} \subset \mathbb{R} P^{m}$. Пара $(X, Y)$ является парой Браудера-Ливси, если вложение $Y \rightarrow X$ индуцирует изоморфизм фундаментальных групп (см. [1], [2]).

Для пары Браудера-Ливси имеет место коммутативная диаграмма точных последовательностей (см. [3], [4]):

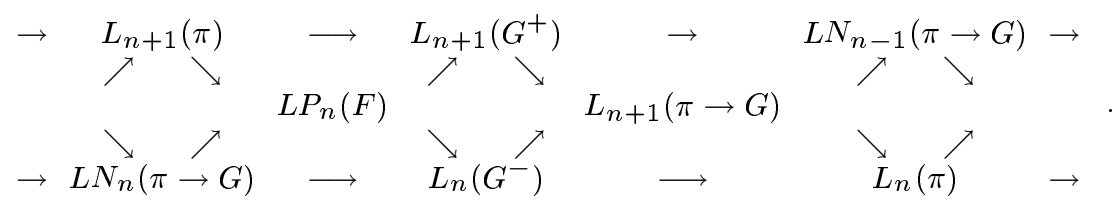

В диаграмму (1) входят группы препятствий к перестройкам $L_{*}(\pi)$ и $L_{*}(G)$, группы $L N_{*}(\pi \rightarrow G)$ препятствий к расщеплению вдоль одностороннего подмногообразия $Y \subset X$ и группы $L P_{*}(F)$ препятствий к перестройкам по паре многообразий $(X, Y)$ (см. [5], [6]).

В работе [7] построена спектральная последовательность в теории перестроек. Для построения используется реализация коммутативной диаграммы (1) на уровне спектров. Основная филштрация спектров из работы [7]

$$
\cdots \rightarrow X_{3,0} \rightarrow X_{2,0} \rightarrow X_{1,0} \rightarrow X_{0,0} \rightarrow X_{-1,0} \rightarrow \cdots
$$

содержит $\mathbb{L}$-спектр $X_{0,0}=\mathbb{L}\left(G^{+}\right)$и спектр $X_{1,0}=\Sigma \mathbb{L} P(F)$ (см. [8], [9]).

Множество $\mathscr{S}_{n}(X, Y, \xi)$ s-триангуляций пары многообразий $(X, Y)$ входит в точную последовательность

$$
\cdots \rightarrow \mathscr{S}_{n}(X, Y, \xi) \rightarrow[X, G / T O P] \stackrel{v_{\xi}}{\rightarrow} L P_{n-1}(F) \rightarrow \cdots
$$

которая может быть построена на уровне спектров [5].

Пусть L• обозначает односвязное накрытие $\Omega$-спектра $\mathbf{L} \bullet(\mathbb{Z})[5],[6]$. При этом для замкнутого топологического многообразия $X$ имеет место изоморфизм $[X, G / T O P] \cong H_{n}(X, \mathbf{L} \bullet)$. Существует также $\Omega$-спектр $\mathbb{S}(X), n$-мерные гомотопические группы которого задают множество топологических триангуляций $\mathscr{S}_{n}(X)$ многообразия $X$.

Пусть $(Z \subset Y \subset X)$ - тройка многообразий, так что каждая из пар многообразий $(Z \subset Y)$, $(Y \subset X)$ является парой Браудера-Ливси. Будем предполагать, что размерность подмногообразия $Z$ не менее 5 .

Будем говорить, что нормалное отображение $f$ перестраивается до простой гомотопической эквивалентности троек многообразий, если класс нормального кобордизма отображения $f$ содержит отображение $g$ со следующими свойствами:

1) отображение $\left.g\right|_{X}$ является простой гомотопической эквивалентностью;

2) отображение $g$ трансверсально подмногообразиям $Y$ и $Z$;

3) ограничения $g$ на трансверсальные прообразы $Y, X \backslash Y, Z, Y \backslash Z$ являются простыми гомотопическими эквивалентностями.

Работа первого автора выполнена при поддержке Российского фонда фундаментальных исследований (грант № 99-01-00009).

Работа второго автора выполнена при поддержке Министерства науки и техники Республики Словения (грант № J1-0885-0101-98). 
Обозначим через $\alpha$ композицию отображений (см. [5])

$$
\mathscr{S}_{n}(X, Y, \xi) \rightarrow \mathscr{S}_{n-1}(Y) \rightarrow L N_{n-2}\left(\pi \rightarrow G^{-}\right) .
$$

Теперь композиция отображения $\alpha$ с отображением $L P_{n}(F) \rightarrow \mathscr{S}_{n}(X, Y, \xi)$ из точной последовательности (3) дает отображение $\beta: L P_{n}(F) \rightarrow L N_{n-2}\left(\pi \rightarrow G^{-}\right)$.

Лемма 1. Существует отображение спектров $b: \Omega^{2} \mathbb{L} P(F) \rightarrow \mathbb{L} N\left(\pi \rightarrow G^{-}\right)$, для которого индуцированное отображение гомотопических групп $b_{*}$ совпадает $c$ отображением $\beta$.

Обозначим через $\mathbb{L} T(X, Y, Z) \Omega$-спектр, являющийся гомотопическим кослоем отображения $b$, а через $L T_{n}(X, Y, Z)$ гомотопические группы $\pi_{n}(\mathbb{L} T(X, Y, Z))$.

Теорема 1. Имеет место отображение спектров $\psi: \Omega^{2}\left(X_{+} \wedge \mathbf{L}_{\bullet}\right) \rightarrow \mathbb{L} T(X, Y, Z)$, индуцирующее гомоморфизм $\psi_{*}: H_{n}(X, L \bullet) \rightarrow L T_{n-2}(X, Y, Z)$. Нормальное отображение $[f: M \rightarrow X] \in[X, G / T O P] \cong H_{n}\left(X, L_{\bullet}\right)$ тогда и только тогда перестраивается до простой гомотопической эквивалентности троек многообразий, когда $\psi_{*}(f)=0$.

Теорема 2. Имеет место гомотопическая әквивалентность спектров

$$
\Sigma^{2} \mathbb{L} T(X, Y, Z) \cong X_{2,0},
$$

әде спектр $X_{2,0}$ входит в фильтрацию (2) спектральной последовательности теории перестроек.

СледСтвИЕ. Пусть $F^{-}$обозначает квадрат фундаментальных групп в задаче расщепления для пары Браудера-Ливси $Z \subset Y$. Имеет место коммутативная диаграмма точных последовательностей

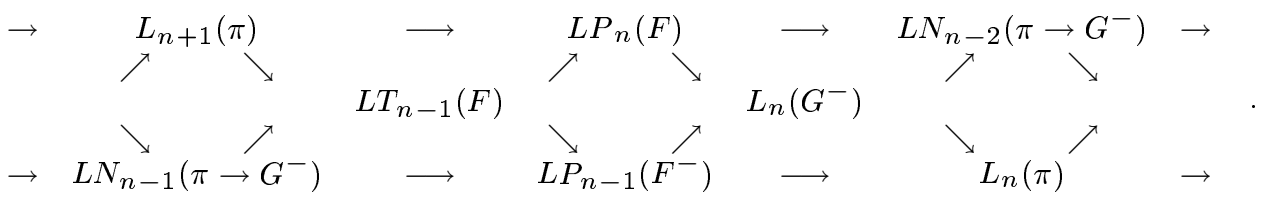

\section{СПИСОК ЛИТЕРАТУРЫ}

[1] W. Browder, G. R. Livesay // Bull. Amer. Math. Soc. 1967. V. 73. P. 242-245. [2] S. E. Cappell, J. L. Shaneson // Lecture Notes in Math. 1979. V. 763. P. 395-447. [3] А. Ф. Харшиладзе // Труды ММО. 1980. Т. 41. С. 3-36. [4] A. A. Ranicki // Canad. J. Math. 1987. V. 39. P. 345-364. [5] A. A. Ranicki. Exact Sequences in the Algebraic Theory of Surgery. Princeton, NJ: Princeton Univ. Press, 1981. (Math. Notes. V. 26.) [6] C. T. C. Wall. Surgery on Compact Manifolds. London: Academic Press, 1970; 2nd ed. Providence, RI: Amer. Math. Soc., 1999. [7] И. Хэмблтон, А. Ф. Харшиладзе // Матем. сб. 1992. Т. 183. № 9. С. 3-14. [8] Ю. В. Муранов, Д. Реповш // Матем. сб. 1997. Т. 188. № 3. С. 127-142. [9] A. Cavicchioli, Yu. V. Muranov, D. Repovš // Boll. Unione Mat. Ital. Sez. B Artic. Ric. Mat. (8). 2001. V. 4. P. 647-675.

Институт современных знаний,

Институт математики, физики и механики Люблянского университета,

Любляна, Словения

E-mail: ymuranov@mail.ru; dusan.repovs@fmf.uni-lj.si 